\title{
SUBFAIR RED-AND-BLACK WITH A LIMIT
}

\author{
DAVID C. HEATH, WILliaM E. PRUITT ${ }^{1}$ AND WILliaM D. SUDDERTH ${ }^{2}$
}

Abstract. Suppose a gambler has an initial fortune in $(0,1)$ and wishes to reach 1 . It is known that, for a subfair red-and-black casino, the optimal strategy is always to bet $\min (f, 1-f)$ whenever the gambler's current fortune is $f$. Furthermore, the gambler should likewise play boldly if there is a house limit $z$ which is the reciprocal of a positive integer; i.e., he should bet $\min (f, 1-f, z)$. We show that if $1 /(n+1)<z<1 / n$ for some integer $n \geqq 3$ or if $z$ is irrational and $\frac{1}{3}<z<\frac{1}{2}$, then bold play is not necessarily optimal.

1. Introduction. Consider the problem faced by a gambler who wishes to obtain at least a certain fortune by wagering at fixed subfair odds on the outcomes of independent repetitions of a random experiment. Coolidge [1] stated:

The player's best chance of winning a certain sum at a disadvantageous game is to stake the sum that will bring him that return in one play, or, if that be not allowed, to make always the largest stake which the banker will accept.

In [2], Coolidge referred to this statement as the "fundamental theorem of games of chance". The proof given by Coolidge is faulty, as was noticed by Dubins and Savage $[3, \S 1.3]$. The conclusion that bold play is optimal is nevertheless correct whenever there is no house limit [3, Chapter 5], and remains valid even if there is a house limit of size $1 / n$th of the desired goal, as was shown by Aryeh Dvoretzky in 1963 (unpublished) and, independently, by Wilkins [4]. The result of this paper goes in the opposite direction and states that for essentially all other limits, bold play need not be optimal.

2. Red-and-black with a house limit. The notation and terminology used below are largely taken from [3].

Received by the editors September 9, 1971 and, in revised form, January 14, 1972. AMS 1970 subject classifications. Primary 60G45.

Key words and phrases. Bold strategy, gambling, optimal strategy.

1 The research of the first two authors was supported by the National Science Foundation under grant GP-28683.

${ }^{2}$ Research was supported by the National Science Foundation under grant GP-24183. 
Let $0<w<\frac{1}{2}, \bar{w}=1-w$, and $z>0$. Consider the gambling problem whose set of fortunes, utility function, and available gambles are as follows: $F=[0,+\infty) ; u(f)=1$ or 0 according as $f \geqq 1$ or $0 \leqq f<1 ; \Gamma_{w, z}(f)=$ $\{w \delta(f+s)+\bar{w} \delta(f-s): 0 \leqq s \leqq \min (f, z)\}$ for all $f \in F$. (Here $\delta(f)$ denotes the probability which assigns mass one to $\{f\}$.)

The gambler stakes $s$ when he uses the gamble $w \delta(f+s)+\bar{w} \delta(f-s)$ at $f$. The bold stake at $f$ is defined by

$$
\begin{aligned}
s(f) & =\min \{f, z, 1-f\}, & & \text { if } 0 \leqq f \leqq 1, \\
& =0, & & \text { if } f>1 .
\end{aligned}
$$

A gambler uses the bold strategy or plays boldly if he stakes $s(f)$ whenever he has fortune $f$.

Let $Q_{w, z}(f)$ be the probability that a gambler starting from $f$ and playing boldly reaches $[1, \infty)$. We often write $Q(f)$ for $Q_{w, z}(f)$ when there is no danger of ambiguity. After the first play, the gambler has $f+s(f)$ or $f-s(f)$ with probability $w$ or $\bar{w}$ respectively. Thus

$$
Q(f)=w Q(f+s(f))+\bar{w} Q(f-s(f))
$$

for all $f$.

By Theorems 2.12.1 and 2.14.1 of [3], the bold strategy is optimal at every $f$ if and only if $Q(f) \geqq w Q(f+s)+\bar{w} Q(f-s)$ for all $f$ and for $0 \leqq s \leqq$ $\min (f, z)$. By [4], the bold strategy is optimal for $z=1 / n, n=1,2, \cdots$.

THEOREM 1. Assume that either (i) $1 /(n+1)<z<1 / n$ for some integer $n \geqq 3$, or (ii) $z$ is irrational and $\frac{1}{3}<z<\frac{1}{2}$. Then there is an $f$ in $(0,1)$ and $a$ number $b$ in $(0, s(f))$ such that, for all positive $w$ sufficiently close to zero,

$$
w Q_{w, z}(f+b)+\bar{w} Q_{w, z}(f-b)>Q_{w, z}(f) .
$$

(That is, the strategy at $f$ which first stakes $b$ and then plays boldly is superior to bold.)

REMARK. The conclusion of the theorem also holds for infinitely many rational $z$ in $\left(\frac{1}{3}, \frac{1}{2}\right)$, as can be seen from the proof. We conjecture that the conclusion holds for all $z$ in $\left(\frac{1}{3}, \frac{1}{2}\right)$.

Proof of Theorem 1. First assume (i). Consider the fortune $f^{*}=$ $[1-(n-1) z] / 2+z$. Notice that

$$
1-(n-1) z+z / 2>f^{*}>1-(n-1) z>z .
$$

Let $k_{0}$ be the largest nonnegative integer $k$ such that $2^{k}<z(1-n z)^{-1}$. Then choose $\varepsilon$ so that $0<\varepsilon<4^{-1}(n z-1)+2^{-\left(k_{0}+2\right)} z$. Notice that (3) remains true when $f^{*}$ is replaced by $f^{*}-2 \varepsilon$. Set $f=f^{*}-\varepsilon, b=z-\varepsilon$, and compare the bold strategy at $f$ to the timid strategy which first stakes $b$ and then plays boldly. After an initial loss, the timid player has $f-b=(1-(n-1) z) / 2$, 
the least fortune from which it is possible to reach 1 in $n$ wins, but the bold player has the strictly smaller fortune $f-z$. Now consider all other ways either gambler can reach 1 with no more than $n$ winning bets. Each can do so with $n-1$ wins in a row; with $n$ wins and one loss at any time after the first win and before the $(n-2)$ win; or with $n-2$ wins in a row followed by a loss, a win, some number of losses not exceeding $k_{0}$, and then a final win. There are no additional ways for either player to reach 1 with $n$ or fewer wins. Moreover, after some finite number $M$ of plays, the bold gambler starting from $f$ must have reached 0 or 1 or have experienced at least $n+1$ winning bets. Hence,

$$
Q_{w, z}(f) \leqq w^{n-1}+(n-2) w^{n} \bar{w}+w^{n} \bar{w}^{2}+\cdots+w^{n} \bar{w}^{k_{0}+1}+2^{M I} w^{n+1} .
$$

Here, $2^{M}$ is an upper bound on the number of paths up to time $M$ and so $2^{M} w^{n+1}$ is an upper bound for the probability that the bold gambler reaches 1 while experiencing at least $n+1$ wins. However, since the timid player can reach 1 with $n$ wins after an initial loss, his return is at least

$$
w^{n-1}+(n-1) w^{n} \bar{w}+w^{n} \bar{w}^{2}+\cdots+w^{n} \bar{w}^{k_{0}+1} .
$$

The conclusion of the theorem follows easily for this case.

Now assume $\frac{1}{3}<z<\frac{1}{2}$ and $z$ is irrational. Choose an integer $k \geqq 2$ so that $(1-z) 2^{-k}+z<1-z$. Let $f^{*}=(1-z) 2^{-k}+z$. Notice that after an initial loss followed by $k$ wins, the bold gambler starting from $f^{*}$ will exactly reach $1-z$. The key to our argument is that he never reaches $1-z$ after an initial win. Some additional notation is needed for the proof.

By a path $P$ is meant a finite sequence of wins and losses. For $f \in[0,1]$, and a path $P$, let $P(f)$ be the fortune of a gambler who starts at $f$, plays boldly, and experiences the sequence $P$ of wins and losses. Let $W$ and $L$ represent the paths of one win and one loss respectively. Then

$$
\begin{aligned}
& W(f)=2 f, \quad 0 \leqq f \leqq z, \quad L(f)=0, \quad 0 \leqq f \leqq z, \\
& =f+z, \quad z \leqq f \leqq 1-z, \quad=f-z, \quad z \leqq f \leqq 1-z, \\
& =1, \quad 1-z \leqq f \leqq 1 ; \quad=2 f-1, \quad 1-z \leqq f \leqq 1 \text {. }
\end{aligned}
$$

LEMMA 1. For every path $P$, the function $f \rightarrow P(f)$ from $[0,1]$ to $[0,1]$ is continuous, monotonic increasing, and piecen'ise linear. Also, every linear segment of $P(f)$ is of the form $a f+b z+c$, where $a, b$, and $c$ are integers.

Proof. Easy by repeated application of (4).

Lemma 2. If $a$ path $P$ begins with a win, then $P\left(f^{*}\right) \neq 1-z$.

Proof. By Lemma 1 and the definition of $f^{*}, P\left(f^{*}\right)=\alpha+\beta z$ where $\alpha$ and $\beta$ are rational. Since $z$ is irrational, $P\left(f^{*}\right)=1-z$ only if $\alpha=1$ and 
$\beta=-1$. If $P$ begins with a win, the coefficient $\beta$ of $z$ is nonnegative however. To see this, notice that after the initial win, the gambler has fortune $2^{-k}+\left(2-2^{-k}\right) z$ so that the coefficient of $z$ is strictly larger than 1 . At the next play, either the coefficient of $z$ becomes and stays zero or it doubles (see (4)). Next the coefficient may be reduced by one, but, if so, it must either double or become permanently zero on the following play. And so forth.

Consider the collection $A$ of all paths $P$ such that the number of wins occurring in $P$ does not exceed $k$ and $1-z<P\left(f^{*}\right)<1$. Notice that the paths along which the bold gambler starting from $f^{*}$ reaches 1 after at most $k+1$ wins are exactly the paths in $A$ followed by a final win.

Lemma 3. $A$ is finite.

Proof. After some finite number of plays the bold gambler starting from $f^{*}$ must have reached 0 or 1 or have experienced at least $k+1$ wins.

Now choose $\varepsilon>0$ so that $z<f^{*}-2 \varepsilon<1-z$ and

$$
P\left(f^{*}-2 \varepsilon\right)>1-z, \text { for every } P \text { in } A .
$$

This is possible by Lemmas 1 and 3. Let $f=f^{*}-\varepsilon$, and $b=z-\varepsilon$. As in the previous case, we compare the bold strategy at $f$ to the timid strategy which first stakes $b$ and plays boldly thereafter. After an initial loss, the timid player has fortune $(1-z) / 2^{k}$, the least fortune from which the goal can be reached in $k+1$ wins, but the bold player has a strictly smaller fortune. After an initial win, the timid player has fortune $f^{*}+z-2 \varepsilon$, the same fortune that a bold player starting from $f^{*}-2 \varepsilon$ would have following an initial win. Thus the timid player's position after experiencing a path $P$ with an initial win is $P\left(f^{*}-2 \varepsilon\right)$. It follows from (5) that every path which reaches 1 in at most $k+1$ wins for the bold player at $f$ will do likewise for the timid. Let $r(w)$ be the probability the bold player reaches 1 with at most $k+1$ wins. Then the bold return is less than or equal to $r(w)+K w^{k+2}$, where $K$ is a constant. However, the timid player's chance of reaching 1 is at least $\bar{w} w^{k+1}+r(w)$. The conclusion of the theorem follows.

Let $U_{w, z}$ be the utility of the gambling house $\Gamma_{w, z}[3, \mathrm{p} .25]$. That is, $U_{u, z}(f)$ is the supremum over all strategies $\sigma$ available at $f$ of the probability under $\sigma$ of reaching 1 .

COROLlary. Let z satisfy condition (i) or (ii) of Theorem 1. Then there is a $\mathrm{W}$ in $\left(0, \frac{1}{2}\right)$ and a nomid open interval $I \subseteq(0,1)$ such that, for every $f \in I$,

$$
Q_{u, z}(f)<U_{u, z}(f)
$$


Proof. By Theorem 1, there exist $f$ and $w$ such that (6) holds. The result follows since $U_{w, z}$ is continuous $[3, \S 4.3]$ and $Q_{w, z}$ is monotonic.

Consider now, for fixed $w$ and $z$, the set $B_{w, z}$ of fortunes in $(0,1)$ at which the bold strategy is optimal. That is, $B_{w, z}=\left\{f \in(0,1): Q_{w, z}(f)=\right.$ $\left.U_{w, z}(f)\right\}$.

Let $B_{z}=\bigcap\left\{B_{w, z}: 0<w<\frac{1}{2}\right\}$. As was mentioned before, if $z=1 / n$, then $B_{z}=(0,1)$. On the other hand, if $z$ satisfies condition (i) or (ii), then, by the corollary, some $B_{w, z}$ excludes an interval. Even if $z \neq 1 / n, B_{z}$ need not be empty. For example, if $\frac{1}{3}<z<\frac{1}{2}$, then $\frac{1}{3} \in B_{z}$. To see this, check that a bold gambler starting from $\frac{1}{3}$ never leaves the set $\left\{0, \frac{1}{3}, \frac{2}{3}, 1\right\}$ and thus his strategy is not affected by the house limit. Hence, $Q_{w, z}\left(\frac{1}{3}\right)=Q_{w, 1 / 2}\left(\frac{1}{3}\right)=$ $U_{w, 1 / 2}\left(\frac{1}{3}\right)$ (since $\left.\frac{1}{3} \in B_{1 / 2}\right) \geqq U_{w, z}\left(\frac{1}{3}\right) \geqq Q_{w, z}\left(\frac{1}{3}\right)$. We have not been able to find elements in $B_{z}$ for any $z$ satisfying condition (i). The next result affirms that, in this case, $B_{z}$ is "small".

THEOREM 2. If $1 /(n+1)<z<1 / n$ for some integer $n \geqq 3$, then, for some $w$ in $\left(0, \frac{1}{2}\right), B_{w, z}$ has Lebesgue measure zero.

Proof. Let $w$ and the interval $I$ be as in the corollary and let $C$ be the complement of $B_{w, z}$ in $(0,1)$. If a gambler starting at $f$ and playing boldly reaches $C$, then it will not be optimal to continue playing boldly and so the bold strategy is not optimal at $f$. In the notation of the proof of Theorem 1 , if, for some path $P, P(f) \in C$, then $f \in C$. It is easy to see that, for some nonempty open interval $J \subseteq(0, z)$ and some path $P, P(J) \subseteq I$. Since $I \subseteq C$, we have $J \subseteq C$.

For each $f \in(0, z)$, we can write $f=z\left(\sum_{k=1}^{\infty} a_{k} 2^{-k}\right)$ where each $a_{k}$ is 0 or 1 . Let $\varphi(f)=f / z=a_{1} a_{2} \cdots$ in binary notation. Define (see equations (4))

$$
\begin{aligned}
S(f) & =W(f), & & \text { if } 0<f<z / 2, \\
& =L(W(f)), & & \text { if } z / 2 \leqq f<z .
\end{aligned}
$$

Then $\varphi(S(f))=a_{2} a_{3} \cdots$ so that $S$ is a "shift operator". Consider the interval $q(J)$. It contains a subinterval of the form $\left\{. a_{1} a_{2} \cdots: a_{1}=\right.$ $\left.b_{1}, \cdots, a_{\Lambda}=b_{N}\right\}$. Since the finite sequence $b_{1} \cdots b_{N}$ occurs somewhere in the binary representation of almost every number in $(0,1)$, we have that, for almost all $f \in(0, z), S^{k} f \in J$ for some $k$ and, hence, $f \in C$.

Finally, for $f \in(z, 1)$, let $m(f)$ be the least integer $m$ such that $L^{m}(f)<z$. Then the function $f \rightarrow L^{m(f)}(f)$ is piecewise continuous and piecewise strictly monotone and so maps almost every $f$ in $(z, 1)$ into $C \cap(0, z)$.

REMARK. We do not know whether Theorem 2 also holds for $z$ in $\left(\frac{1}{3}, \frac{1}{2}\right)$.

So far we have studied the manner in which optimality of bold play depends on the initial fortune $f$ and the house limit $z$. The dependence on the win probability $w$ is also interesting. If $w^{>}>\frac{1}{2}$, then bold play is certainly 
not optimal since, in this case, $U_{w, z}(f)=1$ for all $f[3, \S 4.4]$. Theorem 1 asserts that bold play is sometimes less than optimal for small $w$. It can be shown by an elaborate computation that if $z=2 / 5$ and $f=19 / 40-1 / 640$, then the strategy which stakes $2 / 5-1 / 640$ on the first bet and then plays boldly is better than bold at $f$ for all $w \in\left(0, \frac{1}{2}\right)$.

ACKNowledgment. We are grateful to Lester Dubins for calling the unpublished result of Dvoretzky, as well as certain preliminary work of Sam Shye, to our attention and for suggesting that we study the general problem. We also want to thank Albert Kinderman for a number of helpful conversations.

\section{REFERENCES}

1. J. L. Coolidge, The gambler's ruin, Ann. of Math. 10 (1908/09), 181-192.

2. —, An introduction to mathematical probability, Oxford Univ. Press, Oxford, 1925; republished by Dover, New York, 1962. MR 26 \#6997.

3. Lester E. Dubins and Leonard J. Savage, How to gamble if you must, McGraw-Hill, New York, 1965. MR 38 \#5276.

4. J. Ernest Wilkins, The bold strategy in presence of house limit, Proc. Amer. Math. Soc. 32 (1972), 567-570.

Department of Mathematics, University of Minnesota, Minneapolis, Minnesota 55455 (Current address of David C. Heath and William E. Pruitt)

Department of Statistics, University of Minnesota, Minneapolis, Minnesota 55455 (Current address of William D. Sudderth) 